Revista Destaques Acadêmicos, Lajeado, v. 9, n. 3, 2017. ISSN 2176-3070

DOI: http://dx.doi.org/10.22410/issn.2176-3070.v9i3a2017.1491

www.univates.br/revistas

\title{
A INFLUÊNCIA DO NÍVEL DE ATIVIDADE FÍSICA NO EQUILÍBRIO EM MULHERES IDOSAS
}

\author{
Fabian Arruda Barbosa ${ }^{1}$, Augusto Brugnera ${ }^{2}$, Bruno De Maman ${ }^{3}$, \\ Rômulo Augusto Scherer ${ }^{4}$, Daniela Beatriz da Silva ${ }^{5}$, \\ Jéssica Luana Dornelles da Costa ${ }^{6}$, Caroline Pieta Dias ${ }^{7}$, \\ Carlos Leandro Tiggemann ${ }^{8}$
}

\begin{abstract}
Resumo: O objetivo deste estudo foi avaliar a influência do nível de atividade física no equilíbrio em mulheres idosas. A amostra foi constituída por 15 mulheres idosas, com idade entre 60 e 78 anos, aparentemente saudáveis. Para se avaliar o nível de atividade física foi aplicado o Questionário Internacional de Atividade Física (IPAQ). Após responderem o IPAQ as idosas foram divididas em dois grupos: idosas sedentárias (GSED; $\mathrm{n}=8$ ) e ativas (GATI; $\mathrm{n}=7$ ). Para a avaliação do equilíbrio foram utilizados a Baropodometria, Escala de Equilíbrio de Berg e o teste Time Up and Go. Somente foram encontradas diferenças significativas $(\mathrm{p} \leq 0,05)$ entre os grupos no teste Time Up and Go a favor do grupo GATI $(6,03 \pm 0,48$ vs $6,89 \pm 0,88 \mathrm{~s})$. Concluímos neste estudo que o nível de atividade física tem influência no equilíbrio dinâmico e não no equilíbrio estático de mulheres idosas.
\end{abstract}

Palavras-chave: Nível de atividade física. Idosas. Equilíbrio.

1 Graduado em Educação Física Bacharelado - Univates.

2 Acadêmico do Curso de Educação Física Bacharelado - Univates.

3 Graduado em Educação Física Bacharelado - Univates.

4 Graduado em Educação Física Bacharelado - Univates.

5 Acadêmica do Curso de Educação Física Bacharelado - Univates.

6 Graduada em Educação Física Licenciatura - Univates.

7 Doutora em Ciência do Movimento Humano - Universidade Federal do Rio Grande do Sul (UFRGS).

8 Doutor em Ciência do Movimento Humano - Universidade Federal do Rio Grande do Sul (UFRGS). 


\section{INTRODUÇÃO}

A expectativa de vida da população mundial vem crescendo consideravelmente nos últimos anos, resultando no aumento idosos, sendo que no Brasil seguimos esta tendência de crescimento da população idosa (SIMÕES et al., 2011). Um dos fatores que explica estas mudanças é a melhora dos hábitos de vida das pessoas, ocasionando o aumento da expectativa de vida (SIQUEIRA et al.; 2007; CHAIMOWICZ, 1997).

De acordo com dados obtidos de pesquisas do Instituto Brasileiro de Geografia e Estatística (IBGE), a expectativa de vida média do brasileiro que era de 63 anos em 1980 passou para 78 anos em 2012, um acréscimo de 15 anos (IBGE, 2013). Em 2010 a população de idosos passou a representar $10 \%$ do total da população brasileira, sendo que as projeções para 2030 sugerem que chegará a 19\%. Com este envelhecimento populacional, existe uma maior incidência de doenças crônico-degenerativas, fazendo necessário uma maior preparação e adequação dos serviços de saúde, incluindo a formação e capacitação de profissionais (SIQUEIRA et al., 2007).

O envelhecimento pode ser conceituado de diferentes formas, dentre as quais uma das definições conceitua como a perda da eficiência dos processos morfológicos e funcionais, levando desta forma os idosos a possíveis traumas e doenças (DIAS et al., 2012). Com o aumento da perspectiva de vida os idosos podem sofrer fenômenos funcionais como o da perda no equilíbrio, o que pode prejudicar a prática de tarefas simples, como se levantar de uma cadeira e sair caminhando (SILVA et al., 2006). O equilíbrio corporal quando relacionado com as faixas etárias da população tem grande importância e quando relacionamos com a prática da atividade física na terceira idade está importância aumenta (TEIXEIRA et al., 2008).

As práticas de atividades físicas são primordiais para os idosos, melhorando a qualidade de vida e podendo minimizar os efeitos adversos do processo de envelhecimento (PETERSON; GORDON, 2011). O exercício físico é uma prática de atividade física e quando elaborado pensando no idoso deve implicar em globalidade, incluindo exercícios que se assemelham às atividades diárias, movimentos generalizados e mecanismos de progressão (PEREIRA, 2009).

Idosos que participam regularmente em programas estruturados de exercícios físicos, apresentam melhor mobilidade funcional, equilíbrio e menor risco de queda do que idosos não treinados (RIBEIRO et al., 2008). O equilíbrio pode ser mantido ou até melhorado com a idade através da prática de exercícios de treinamento específico para equilíbrio (ACSM, 2011). Diversos estudos fazem uso de diferentes testes, como por exemplo, a Baropodometria (ALFIERI; TEODRI; GUIRRO, 2006), a Escala de equilíbrio de Berg (EEB) (SANTOS et al., 2016) e o teste Time Up and Go (TUG) para avaliar o equilíbrio, 
tentando relacionar a influência da prática habitual de atividade física com a melhora desta variável em idosos (TIGGEMANN et al., 2016).

Estudos têm evidenciado que idosos praticantes de musculação, com uma frequência regular de pelo menos duas vezes por semana, conseguem obter como resultado uma melhora na força e equilíbrio dinâmico (TIGGEMANN et al., 2016; SOUZA et al., 2014). Contudo, pouco tem se investigado sobre o efeito das atividades físicas habituais sobre o equilíbrio. Desta forma, o objetivo deste estudo foi verificar a influência do nível de atividade física no equilíbrio em mulheres idosas.

\section{MÉTODO}

A pesquisa se classifica como um estudo transversal quantitativo, tendo sido aprovada pelo Comitê de Ética em Pesquisa (COEP) da Univates (parecer número 1.567.120). Sendo entregue às participantes um Termo de Consentimento Livre Esclarecido (TCLE), do qual estavam cientes do estudo e concordaram em participar da pesquisa de sua livre e espontânea vontade.

A amostra foi composta de 15 mulheres com idade entre 60 e 78 anos, aparentemente saudáveis, recrutadas através de meios de comunicação, como jornal, rádio e folders sobre o estudo. Foi adotado como critério de inclusão para participação do estudo, os sujeitos que (1) não tenham praticado treinamento de força pelo mínimo 6 meses antes do começo do estudo; (2) apresentassem ausência de histórico de doenças cardiovasculares (a exceção de hipertensão arterial controlada por medicamento), endócrinas, metabólicas, neuromusculares e articulares (a exceção de quadros leves de artrite e/ou artrose); e (3) não estar em tratamento à base de medicamentos de uso contínuo ou eventual que afetasse o equilíbrio.

A amostra foi caracterizada quanto o nível de atividade física por meio da aplicação do Questionário Internacional de Atividade Física (IPAQ, 2005). O IPAQ foi desenvolvido para avaliar os níveis da prática de atividade física de diversas populações, que é culturalmente adaptável, sem perder suas especificidades. Ele mede o tempo gasto em diferentes atividades físicas, com um tempo mínimo de dez minutos e os sujeitos podem ser classificados em três níveis de atividade física: baixo, moderado e intenso, conforme os dados de uma semana habitual (IPAQ, 2005). O questionário foi aplicado individualmente em entrevista no Laboratório de Fisiologia do Exercício da Univates.

Os testes de equilíbrio foram realizados nas dependências do complexo esportivo da Univates, no turno da tarde, e após uma semana, os mesmos foram replicados (reteste), visando minimizar os erros de medida. Todos os testes foram realizados por avaliadores aptos e devidamente treinados. Já a avaliação do equilíbrio consistiu de três testes físicos específicos, sendo eles a Baropodometria, Escala de Equilíbrio de Berg (EEB), Time Up and Go (TUG). 
A Baropodometria Estática foi realizada através do Baropodômetro Eletrônico, que documenta as análises com imagens de pontos de pressão plantar medidos e acaba armazenando todas as informações adquiridas a nível estático, verificando a postura do indivíduo na posição estática, bem como as disfunções funcionais do equilíbrio e estabilidade postural, sendo as aquisições das imagens são precisas, instantâneas, repetíveis e não invasivas. A Baropodometria Estática fornece o valor da superfície plantar das idosas por centímetros quadrados $\left(\mathrm{cm}^{2}\right)$ (BANKOFF et al., 2007). O teste foi aplicado com a idosa na posição estática, com as pernas afastadas com os pés na linha do quadril, no primeiro momento com os olhos abertos (PAOA) e depois com os olhos fechados (PAOF). O teste foi executado uma vez em cada idosa, tendo duração de 50 segundos, com um recorte de 30 segundos para análise.

Referente ao equilíbrio funcional, utilizou-se o teste Escala de Equilíbrio de Berg (EBB), com base em 14 itens comuns para a vida cotidiana, como por exemplo sentar e levantar de uma cadeira. A pontuação máxima a ser alcançado nesta escala é 56 e cada item tem uma escala ordinal que consiste em cinco opções variando de 0 a 4 pontos de acordo com o nível de dificuldade (quanto maior a pontuação, melhor o equilíbrio). O teste é de simples execução, fácil administração e seguro para a avaliação de sujeitos idosos (BERG et al., 1989).

O teste Time Up and Go (TUG) tem início a partir da posição sentada em uma cadeira (43 $\mathrm{cm}$ de altura, com costas apoiadas), e consiste em as idosas levantaram da mesma (sem auxílio das mãos), caminharam e contornaram um cone posicionado no solo a 2,43 m de distância e voltar a se sentar (com as costas apoiadas). Duas tentativas foram feitas em cada uma das velocidades: máxima e habitual, com intervalo de 3 minutos entre cada tentativa, sendo registrado menor tempo de cada velocidade (TIGGEMANN et al., 2016). O TUG se mostra uma ferramenta útil para a previsão de nível de mobilidade funcional dos idosos (SHUMWAY-COOK; BRAUER; WOLLACOTT, 2000).

Os dados foram apresentados por meio da estatística descritiva (média \pm desvio padrão). A normalidade dos dados foi testada por meio do teste de Shapiro-Wilk e a homogeneidade pelo teste de Levene. A comparação das médias das variáveis entre os grupos foi testada por meio do teste $t$ independente. Um nível de significância de $\mathrm{p} \leq 0,05$ foi utilizado, por meio do pacote estatístico SPSS v. 18.0.

\section{RESULTADOS}

A caracterização da amostra e os resultados comparativos estão apresentados na tabela 1 , onde somente os níveis de atividade física $(\mathrm{p}<0,001)$ e o teste TUG $(\mathrm{p}=0,039)$ apresentaram diferenças significativas entre os grupos. Nas demais variáveis do estudo (idade, massa corporal, estatura, PAOA, PAOF e EEB) não foram constatadas diferenças significativas entre os grupos. 
Tabela 1. Estatística descritiva (média e desvio padrão) do grupo sedentárias $($ GSED $n=8)$ e do grupo ativas (GATI n=7)

\begin{tabular}{l|c|c|c}
\hline Variáveis & GSED $(\mathrm{n}=8)$ & GATI $(\mathrm{n}=7)$ & $\mathrm{p}$ \\
\hline Idade $($ anos) & $66,25 \pm 6,52$ & $63,00 \pm 2,89$ & 0,246 \\
\hline Massa Corporal $(\mathrm{Kg})$ & $72,44 \pm 13,33$ & $66,23 \pm 11,34$ & 0,353 \\
\hline Estatura $(\mathrm{m})$ & $1,57 \pm 0,06$ & $1,52 \pm 0,05$ & 0,093 \\
\hline $\begin{array}{l}\text { Nível de Atividade Física } \\
\text { (METS/min/sem) }\end{array}$ & $353,00 \pm 194,80$ & $1292,43 \pm 306,94$ & $<0,001^{*}$ \\
\hline PAOA $\left(\mathrm{cm}^{2}\right)$ & $0,90 \pm 0,20$ & $0,92 \pm 0,44$ & 0,899 \\
\hline PAOF $\left(\mathrm{cm}^{2}\right)$ & $1,55 \pm 1,04$ & $1,89 \pm 1,7$ & 0,643 \\
\hline EEB $(\mathrm{pt})$ & $52,75 \pm 2,31$ & $54,71 \pm 0,76$ & 0,052 \\
\hline TUG $(\mathrm{seg})$ & $6,89 \pm 0,88$ & $6,03 \pm 0,48$ & $0,039^{*}$ \\
\hline
\end{tabular}

GSED = grupo sedentárias; GATI = grupo ativas; PAOA = Baropodometria Estática Pés Afastados Olhos Abertos; PAOF = Baropodometria Estática Pés Afastados Olhos Fechados; EEB = Escala de Equilíbrio de Berg; TUG = Time Up and Go. *diferença significativa entre grupos $(p \leq 0,05)$.

\section{DISCUSSÃO}

O presente estudo teve como finalidade verificar a influência do nível de atividade física no equilíbrio de mulheres idosas, sendo evidenciada diferença significativa apenas no teste TUG, onde as idosas ativas realizaram o teste em menor tempo que as sedentárias. A prática regular de atividade física pode vir a exercer melhoras significativas na manutenção do equilíbrio corporal. Além disso, pode produzir benefícios na saúde dos idosos e desta forma contribuindo para uma menor incidência de quedas e possíveis traumas nessa população (MAZO et al., 2007; RESENDE; RASSI; VIANA, 2008; MANN et al., 2009).

Estudos já corroboram que mulheres possuem uma maior prevalência de quedas decorrentes da perda de equilíbrio na fase do envelhecimento do que os homens, decorrente de fatores como a osteoporose e a sarcopenia (BERNARDI et al., 2008; FROST, 2008). As quedas podem afetar negativamente a qualidade de vida destas pessoas, da mesma forma como a expectativa de vida. Sendo assim, a prática regular de atividade física pode contribuir na melhora do equilíbrio e na prevenção de quedas (RIBEIRO; PEREIRA, 2005).

No que se refere ao teste de Baropodometria Estática, os achados deste estudo não mostraram diferença significativa entre os grupos avaliados. Estes resultados não corroboram com os resultados apresentados por Alfieri, Teodori e Guirro (2006), o qual observou que a prática regular de exercício físico tem influência no equilíbrio postural de idosas entre 60 a 70 anos. No entanto, Rubira et al. (2014) avaliaram 15 idosos saudáveis de ambos os sexos entre 65 e 70 anos, se assemelhando com a população do presente estudo, não 
ficando evidenciado que a prática regular de exercício físico tem influência no equilíbrio estático.

Também Famula et al. (2013), após avaliarem 62 idosas através do tapete de equilíbrio com os olhos abertos e fechados, não encontraram diferença significativa entre ser ativo ou sedentário na melhora do equilíbrio. Um dos fatores que pode ter influenciado no resultado do estudo segundo os autores, é que ambos os grupos de idosas eram ativas na fase jovem ou adulta, sendo sugerido que este fato possa ter alguma relação com o bom equilíbrio no envelhecimento. Embora no nosso estudo o nível de atividade física em outras fases da vida não tenha sido avaliado, isso também poderia explicar nossos achados.

Em relação ao teste da EEB, nossos resultados não apresentaram diferenças significativas entre os grupos. Uma possível explicação para isto é que, por se tratar de mulheres saudáveis, ambos os grupos quase atingiram a pontuação máxima do teste, sendo uma hipótese para os resultados a limitação da EEB, o qual possui pontuação máxima de 56 pontos. Esta hipótese também é sugerida por Ribeiro e Pereira (2005), onde 30 idosas foram avaliadas pela EEB, sendo que os autores consideraram esta escala ser um instrumento frágil para detectar alterações no equilíbrio em idosos saudáveis.

No presente estudo, constatou-se que as idosas ativas obtiveram melhor tempo que as sedentárias no teste de TUG, apresentando assim, um melhor equilíbrio dinâmico. Corroborando com o achado Tomicki et al. (2016), que em seu estudo com uma amostra de 30 idosos, divididos em um grupo controle e um grupo intervenção, os idosos do grupo intervenção praticaram exercício físico três por semana pelo período de três meses, e o grupo controle praticou somente suas atividades de vida diária, o que não os qualificava como ativos. Evidenciando assim, que o grupo intervenção obteve melhores resultados no TUG, indicando uma melhora no equilíbrio dinâmico em indivíduos ativos.

Para Figueiredo, Lima e Guerra (2007), o teste TUG possui grande aceitação na literatura científica, sendo de fácil aplicação e compreensão, podendo avaliar a mobilidade funcional e o equilíbrio em idosos. Estudo como o de Guimarães et al. (2004), o qual avaliou 40 idosos, 20 ativos e 20 sedentários, ficou evidenciado que idosos ativos possuem uma menor propensão a quedas, melhoram o equilíbrio e a capacidade de realizar tarefas simples do cotidiano, apresentam melhores escores no TUG. De acordo com Freitas et al. (2013), a prática habitual de atividade física melhora o equilíbrio corporal, corroborando com os resultados obtidos nesta pesquisa.

Possivelmente o fato de apenas um teste ter encontrado diferença entre os grupos deste estudo, pode ter uma provável relação com o fator do tipo de equilíbrio avaliado. De acordo com Souza et al. (2016), o teste TUG serve para medir o equilíbrio dinâmico, usando mudanças da posição sentada para em pé, podendo avaliar a estabilidade e trocas de direção na caminhada, sem o uso de estratégias que possam alterar estes movimentos. Desta forma indivíduos 
ativos podem vir a obter um melhor resultado neste teste. A EEB, assim como a Baropodometria Estática, por se tratarem de testes que avaliam o equilíbrio estático, parecem ser protocolos limitados para avaliação do equilíbrio entre idosos ativos e sedentários aparentemente saudáveis.

\section{CONCLUSÃO}

Como conclusão deste estudo, pode-se constatar que o nível de atividade física tem influência no equilíbrio dinâmico e não no equilíbrio estático de mulheres idosas saudáveis. Sendo considerado como uma das limitações deste estudo, o número reduzido de idosas que participaram dos testes, se fazendo necessário para possíveis estudos futuros uma maior população amostral. Também é sugerido estudos semelhantes em idosos de mais idade ou ainda, um maior comprometimento físico.

\section{REFERÊNCIAS}

ALFIERI, Fábio M.; TEODORI, Rosana M.; GUIRRO, Rinaldo R. J. de; Estudo baropodométrico em idosos submetidos à intervenção fisioterapêutica. Fisioterapia em Movimento, v. 19, n. 2, p. 67-74, 2006.

American College of Sports Medicine Diretrizes do ACSM para os testes de esforço e sua prescrição. Ed 9 - Rio de Janeiro: Guanabara, 2011.

BANKOFF, Antonia D. P. et al. Estudo do equilíbrio corporal postural através do sistema de baropodometria eletrônica. Revista conexões, v. 2, n. 2, p. 87-104, 2007.

BERG, K. O. et al. Measuring balance in the elderly: preliminary development of an instrument. Physiotherapy Canada, v. 41, n. 6, p. $304-311,1989$.

BERNARDI, Daniela F. et al. O tratamento da sarcopenia através do exercício de força na prevenção de quedas em idosos: revisão da literatura. Ensaios e Ciência: Ciências Biológicas, Agrárias e da Saúde, v. 7, n. 2, 2008.

CHAIMOWICZ, Flávio. A saúde dos idosos brasileiros às vésperas do século XXI: problemas, projeções e alternativas. Revista de Saúde Pública, v. 31, n. 2, p. 184-200, 1997.

DIAS, Caroline et al. Relação entre os níveis de atividade física e qualidade de vida de idosos sedentários e fisicamente ativos. Revista Brasileira Geriatria e Gerontologia, v. 15, n. 4, p. 635-642, 2012.

FAMULA, A. et al. Previous physical activity and body balance in elderly people. Journal of Biology of Sport, v. 30, n. 4, p. 311-315, 2013. 
FIGUEIREDO, Karyna M. O. B. de; LIMA, Kênio C.; GUERRA, Ricardo O. Instrumentos de avaliação do equilíbrio corporal em idosos. Revista Brasileira de Cineantropometria e Desempenho Humano, v. 9, n. 4, p. 408-413, 2007.

FREITAS, Elaine R. F. S. et al. Prática habitual de atividade física afeta o equilíbrio de idosas? Fisioterapia e Movimento, v. 26, n. 4, p. 813-821, 2013.

FROST, Harold M. Osteoporosis: a rationale for further definitions? Calcified tissue international, v. 62, p. 89-94, 1998.

GUIMARÃES, Laiz H. C. T. et al. Comparação da propensão de quedas entre idosos que praticam atividade física e idosos sedentários. Revista Neurociências, v. 12, n. 2, 2004.

Instituto Brasileiro de Geografia e Estática - IBGE. Projeção da população. 2013.

Disponível em: http:/ / www.ibge.gov.br/home/estatistica/populacao/projecao_da_ populacao/2013/default_tab.shtm>. Acesso em 13 março 2016.

IPAQ (2005). Guidelines for Data Processing and Analysis of the International Physical Activity Questionnaire. Disponível em: <http:/www.ipaq.ki.se/>. Acesso em 05 março 2016.

MANN, Luana et al. Equilíbrio corporal e exercícios físicos: uma revisão sistemática. Revista Motriz, v. 15 n. 3 p .713-722, 2009.

MAZO, G. Z. et al. Condições de saúde, incidência de quedas e nível de atividade física dos idosos. Revista Brasileira de Fisioterapia, v. 11, n. 6, p. 437-442, 2007.

PEREIRA, Cássio A. Treinamento de Força Funcional: desafiando o controle postural. $1^{\text {a }}$ edição. Jundiaí/SP: Ed. Fontoura, 2009.

PETERSON, M. D.; GORDON, P. M.; Resistance exercise for the aging adult: clinical implications and prescription guidelines. The American Journal of Medicine, v. 124, n. 3, p. 194-198, 2011.

RESENDE, S. M.; RASSI, C. M.; VIANA, F. P.; Efeitos da hidroterapia na recuperação do equilíbrio e prevenção de quedas em idosas. Revista Brasileira Fisioterapia, v. 12, n. 1, p. 57-63, 2008.

RIBEIRO, Angela S. B.; PEREIRA, João S. Melhora do equilíbrio e redução da possibilidade de queda em idosas após os exercícios de Cawthorne e Cooksey. Revista Brasileira Otorrinolaringologia, v. 71, n. 1, p. 38-46, 2005.

RIBEIRO, Adalgysa P. et al. A influência das quedas na qualidade de vida de idosos. Ciência e Saúde Coletiva, v. 13, n. 4, p.1265 - 1273, 2008

RUBIRA, Ana P. F. A. et al. Efeito de exercícios psicomotores no equilíbrio de idosos. ConScientiae Saúde, v. 13, n. 1, p. $54-61,2014$. 
SANTOS, Isabela R. et al. Análise dos parâmetros da marcha e do equilíbrio dos idosos após exercícios aeróbicos e terapêuticos. Arquivos Ciência e Saúde UNIPAR, v. 20, n. 1, p. 19-23, 2016.

SHUMWAY-COOK, Anne; BRAUER, Sandy; WOLLACOTT, Marjorie. Predicting the probability for falls in community-dwelling older adults using the Timed Up \& Go Test. Journal of the American Physical therapy Association, v. 80, n. 9, p. 896-903, 2000 .

SILVA, Tatiana A. A. et al. Sarcopenia Associada ao Envelhecimento: Aspectos Etiológicos e Opções Terapêuticas. Revista Brasileira de Reumatologia, v. 46, n.6, p. 391-397, 2006.

SIMÕES, Roberto et al. Treinamento de força para idosos. Cooperativa fitness, 2011.

SIQUEIRA, Fernando V. et al. Prevalência de quedas em idosos e fatores associados. Revista de Saúde Pública. v. 41, n. 5, p. 749-756, 2007.

SOUZA, Lucas K. de et al. Comparação dos níveis de força e equilíbrio entre idosos praticantes de musculação e de hidroginástica. Revista Brasileira de Atividade Física e Saúde, v. 19, n. 5, p. 647 - 648, 2014.

TEIXEIRA, Clarissa S. et al. Equilíbrio corporal e exercícios físicos: uma investigação com mulheres idosas praticantes de diferentes modalidades. Acta Fisiátrica, v. 15, n. 3, p. 156-159, 2008.

TIGGEMANN, Carlos L. et al. A percepção de esforço no treinamento de força. Revista Brasileira de Medicina do Esporte. v. 16, n. 4, p. 301-309, 2010.

TIGGEMANN, Carlos L. et al. Effect of traditional resistance and power training using rated perceived exertion for enhancement of muscle strength, power, and functional performance. The Official Journal of the American Aging Association, v. 38, n. 42, p. 1-12, 2016.

TOMICKI, Camila et al. Efeito de um programa de exercícios físicos no equilíbrio e risco de quedas em idosos institucionalizados: ensaio clínico randomizado. Revista Brasileira de Geriatria e Gerontologia, v. 19, n. 3, p. 473-482, 2016. 